\title{
Anesthesia for endoscopic retrograde cholangiopancreatography: target-controlled infusion versus standard volatile anesthesia
}

\author{
Youssef Motiaa, Mustapha Bensghir, Abdelhamid Jaafari, Mohammed Meziane, Redouane Ahtil, \\ Noureddine Drissi Kamili \\ Mohamed V Military Hospital of Instruction, Mohamed V University, Morocco
}

\section{Abstract}

\section{Introduction}

Endoscopic retrograde cholangiopancreatography (ERCP) is the gold standard in the diagnosis and treatment of biliary and pancreatic disease [1]. In France, about 45,000 patients underwent this technique [2]. The use of this technique has a diagnostic and therapeutic interest, as it is indicated for patients

Department of Anesthesiology and Critical Care, Mohamed V Military Hospital of Instruction, Faculty of Medicine and Pharmacy of Rabat, Mohamed V. University, Morocco

Conflict of Interest: None

Correspondence to: Youssef Motiaa, MD, Department of Anesthesia and Critical Care, Mohammed V Military University Hospital,

Faculty of Medicine and Pharmacy, Mohamed V University,

Rabat, Morocco, Tel.: +212 0671195295,

e-mail: youssefmotiaa@gmail.com

Received 27 March 2016; accepted 12 June 2016;

published online 14 July 2016

DOI: http://dx.doi.org/10.20524/aog.2016.0071 with co-morbidities [3]. In fact, with the wide use of ERCP, many critical patients in whom conventional surgery was a high-risk procedure could be managed by endoscopic treatment.

Because of patient's status and procedures difficulties, the choice of the anesthetic technique presents a real challenge. Many techniques have been proposed [4-9] with sedation used mostly [7-9]. However, it carries some risks [10-14], and the use of general anesthesia may be necessary in some patients. Because of co-morbidities in patients undergoing ERCP, the optimization of general anesthesia for this technique is necessary. We thus conducted a prospective study to compare two anesthetic protocols: general anesthesia versus intravenous anesthesia target controlled.

\section{Patients and methods}

The study protocol was approved by the local ethics committee of the Mohammed V Military Hospital at Rabat. All patients scheduled for elective ERCP between January and 
June 2011 were recruited in the study and provided written informed consent. Exclusion criteria were: allergy to iodinated contrast agents; propofol or morphine; age less than 18 years; chronic use of morphine; pregnancy; full stomach; difficult intubation criteria; and patients undergoing emergency ERCP and who required surgical conversion for intra-operative complication. All ERCPs were conducted by the same team of anesthesiologists and endoscopists.

For each included patient, demographic factors [age, sex, body mass index (BMI)], ASA classification, diagnosis, and endoscopic intervention were noted. Patients included were randomized into two groups according to anesthetic protocol: standard anesthesia (Gr: SA), and target-controlled infusion (Gr: TCI) using sealed envelopes.

\section{Anesthetic protocol}

In the pre-anesthetic consultation, all chronic treatments were managed as recommended. After a fasting period of $6 \mathrm{~h}$ and premedication with hydroxyzine $(1 \mathrm{mg} / \mathrm{kg})$ the night before and the morning of procedure, patients were taken to the operating room. The anesthesiologist opened the sealed envelope and prepared the anesthetic technique as indicated. All patients were continuously monitored for heart rate (by using a three-lead electrocardiogram), blood pressure (by using an automated blood pressure cuff and serial measurements every $5 \mathrm{~min}$ ), oxygen saturation (by pulse oximetry) and capnography. Vascular access was secured in all patients via peripheral vein and infusion of $0.9 \%$ saline $(5 \mathrm{~mL} / \mathrm{kg})$ was started. In addition, a dose of $2 \mathrm{~g}$ of amoxicillin and clavulanic acid was administered prior to the procedure.

After 3 min of pre-oxygenation and expired oxygen fraction superior to $92 \%$, anesthesia was induced in both groups:

- Gr: SA: induction was done by fentanyl (1-2 $\mu \mathrm{g} / \mathrm{kg})$ and propofol (1.5-2 mg/kg) and maintenance was performed by sevoflurane (1-1.5\%) and $50 \%$ mix of $\mathrm{N}_{2} \mathrm{O}$ and oxygen.

- Gr: TCI: induction and maintenance were done with propofol and remifentanil. For induction, propofol $(10 \mathrm{mg} / \mathrm{mL})$ was started with the Schnider model with an initial target of $0.5 \mathrm{ng} / \mathrm{mL}$. For remifentanil $(50 \mu \mathrm{g} / \mathrm{mL})$, it was administered by the Minto model with an initial target of $0.75 \mathrm{ng} / \mathrm{mL}$. Maintenance of anesthesia was done by the same drugs in TCI mode.

In all patients, Muscle relaxation was achieved with cisatracurium $(0.15 \mathrm{mg} / \mathrm{kg})$. Variation less than $30 \%$ of the mean arterial pressure (MAP) and heart rate (HR) was considered as hemodynamic objectives.

\section{Study design}

The primary endpoint of the study was extubation timing between Gr: SA and Gr: TCI. Peri-operative hemodynamic and respiratory complications, needs of antispasmodic, overall satisfaction by endoscopist, and stay in the recovery room were compared.
MAP and HR were recorded just before and during induction, and continually every $5 \mathrm{~min}$. In cases of hypotension, measurements were made every minute until stabilization of MAP. Oxygen saturation was continually monitored throughout the procedure. At the end of the intervention, target concentrations were set to zero in the Gr: TCI, sevoflurane and nitrous oxide were arrested in the Gr: SA with increasing fresh gas flow to $10 \mathrm{~L} / \mathrm{min}$. The extubation timing was defined as the time between the end of the intervention and the patient extubation according to the recommended criteria. The hemodynamic variations of HR and MAP were defined by any variation over 30\% compared to reference values. Hypotension was treated by solution infusion and administration of vasoconstrictors (ephedrine: bolus of $3 \mathrm{mg} / \mathrm{mL}$ ). Hypertension was treated by increasing the sedation depth with propofol bolus $(20 \mathrm{mg})$ and/or fentanyl $(25 \mu \mathrm{g})$ in the $(\mathrm{Gr}: \mathrm{SA})$ and by increasing the target propofol $(0.1 \mu \mathrm{g})$ and remifentanil $(0.1 \mathrm{ng})$ in the (Gr: TCI). Desaturation was defined as $\mathrm{SpO}_{2}$ less than $95 \%$. HR, MAP and $\mathrm{SpO}_{2}$ values before induction were taken as reference values.

The intravenous administration of antispasmodic: phloroglucinol $(80 \mathrm{mg})$ was made at the endoscopist request. The satisfaction of the endoscopy team was judged on three classes: low, medium and high satisfaction. Thirty minutes before the end of procedure, analgesia was provided by paracetamol (1 g) and intravenous infusion of nefopam (20 mg).

All patients were extubated in the operating room, and were admitted to the post-anesthesia care unit (PACU), where they were monitored (electrocardiogram, $\mathrm{SpO}_{2}$, noninvasive blood pressure) and they received intranasal supplemental oxygen ( $2 \mathrm{~L} / \mathrm{min}$ ). Patients left PACU when their vital signs were within normal range.

\section{Statistical analysis}

In a pilot study (performed in our institution) a difference of $10 \pm 5$ min was noted between the two groups. Based on this study, we considered that a reduction by $33 \%$ in extubation time was important to show the difference [with an error $(\alpha)$ of $5 \%$ and power $(\beta)$ of $80 \%$ ], the number of patients to include was estimated at 36 per group. To increase the power of the study and reduce excluded patients during the study, we decided to include 45 patients per group. Statistical analysis was made by SPSS for Windows, version 13 (SPSS, Inc, Chicago, IL, USA). The chi-square test or Fisher's exact test were used to compare categorical data when appropriate. Student's $t$-test was used for continuous data. A P value $<0.05$ was considered statistically significant.

\section{Results}

Between January 2011 and June 2011, 110 patients eligible for inclusion were selected. 20 patients were excluded and 90 were included with 45 in each group (Fig. 1: flow diagram). 
No significant differences were noted between the two groups regarding age, sex, BMI, ASA classification or diagnosis (Table 1). Extubation time was shorter in Gr: TCI with a mean time of $15 \pm 2.6$ than $27.4 \pm 7.1$ min in Gr: SA $(\mathrm{P}<0.001)$. The incidence of hypotension was higher in Gr: SA than Gr: TCI $(\mathrm{P}=0.009)$. Endoscopist's satisfaction was better in Gr: TCI than Gr: SA ( $\mathrm{P}=0.003)$. In Gr: TCI, intraoperative targets ranged between 0.5 and $2 \mathrm{ng} / \mathrm{mL}$ for propofol and between 0.75 and $2 \mathrm{ng} / \mathrm{mL}$ for remifentanil. Need for antispasmodics

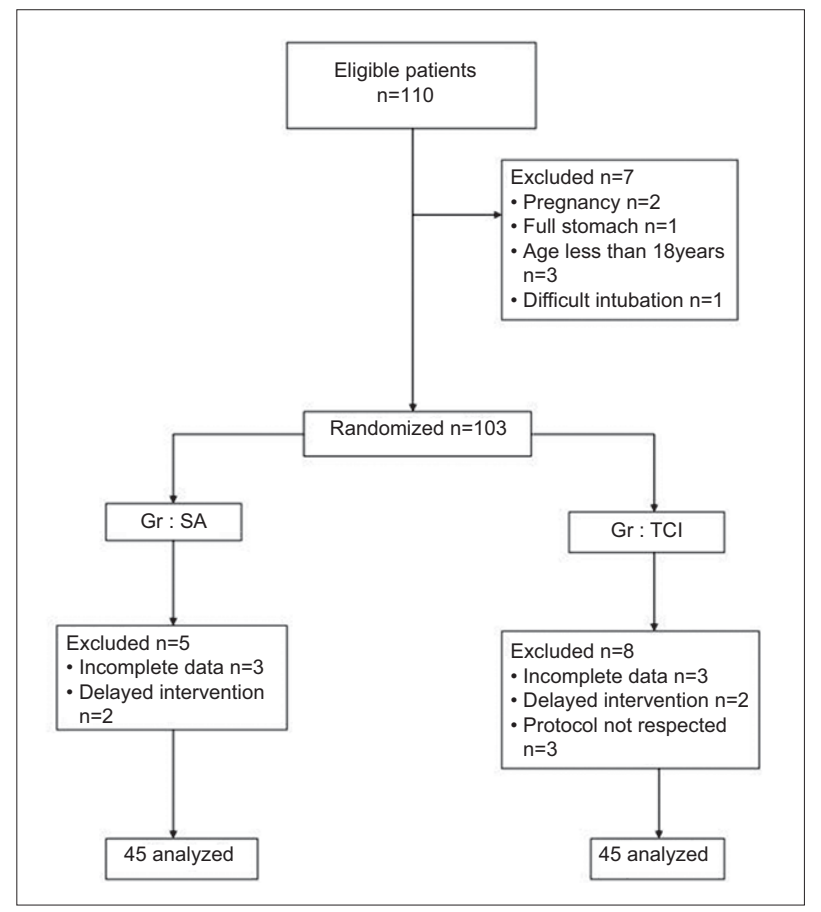

Figure 1 Flow diagram of the process of study evaluation

Table 1 Population characteristics and endoscopic retrograde cholangiopancreatography indications

\begin{tabular}{lccc}
\hline Characteristic & $\begin{array}{c}\text { Group SA } \\
(\mathrm{n}=45)\end{array}$ & $\begin{array}{c}\text { Group TCI } \\
(\mathrm{n}=45)\end{array}$ & $\mathrm{P}$ \\
\hline Age (years) & $58.04 \pm 12.4$ & $53.8 \pm 13.9$ & 0.13 \\
\hline Sex $(\mathrm{M} / \mathrm{F})(\mathrm{n})$ & $22 / 23$ & $16 / 29$ & 0.2 \\
\hline ASA (I/II/III) (n) & $5 / 16 / 24$ & $2 / 13 / 30$ & 0.61 \\
\hline Weight $(\mathrm{kg})$ & $71.3 \pm 4.7$ & $70.3 \pm 5.1$ & 0.35 \\
\hline Height $(\mathrm{cm})$ & $172.1 \pm 3.5$ & $172.4 \pm 3.09$ & 0.75 \\
BMI $\left(\mathrm{kg} / \mathrm{m}^{2}\right)$ & $24.1 \pm 2.4$ & $23.9 \pm 2.9$ & 0.31 \\
\hline Diagnosis $(\mathrm{n})$ & & & 0.56 \\
\hline Pancreatic tumor & 17 & 19 & \\
\hline Bile duct tumor & 11 & 15 & \\
\hline Biliary calculi & 12 & 8 & \\
\hline Other & 5 & 3 & \\
\hline
\end{tabular}

Variables are expressed as mean \pm standard deviation or number. $S A$, standard anesthesia; TCI, target-controlled infusion; $M$, male; F, female; ASA, American Society of Anesthesiologists; BMI, body mass index was higher in Gr: SA than Gr: TCI $(\mathrm{P}=0.023)$. Six patients presented desaturation in the recovery room in $\mathrm{Gr}$ : SA vs a single patient in the Gr: TCI $(\mathrm{P}=0.049)$. The length of the stay in the recovery room was shorter in Gr: TCI than in Gr: SA, with durations of $58.7 \pm 12.4$ and $40.2 \mathrm{~min} \pm 7.3 \mathrm{~min}$ respectively $(\mathrm{P}<0.001)$. One ICU admission for heart failure was needed in the Gr: SA (Table 2); follow up was favorable and the patient was discharged at day two postoperatively.

\section{Discussion}

During ERCP, the use of TCI mode allows better optimization of the general anesthesia, with a shorter extubation time, decreased demand for antispasmodics and less peri-operative complications compared to standard anesthetic technique.

ERCP is the gold standard in the diagnosis and treatment of biliary and pancreatic disease [1]. This technique is often directed to a fragile population and the spectrum of therapeutic applications of ERCP continues to expand, enabling treatment of more complex biliopancreatic disease. The requirement for open surgical and percutaneous techniques has diminished and almost all biliary diseases are now amenable to endoscopic treatment. As a result, many patients previously considered to be inoperable or with life-threatening conditions can now undergo therapeutic ERCP. Martindale et al reported that $46 \%$ of patients who presented for ERCP are ASA physical status 3-5. In such patients, this technique is the optimal treatment of bile duct stones with a success rate of $98 \%$, and improves their quality of life with few associated postoperative complications for neoplastic disease of the bile duct [3].

Anesthesia and analgesia are important elements for the realization of interventional endoscopic procedures. They reduce pain, discomfort and stress for patients and contribute to improved tolerance, patient comfort and success of procedure with the least morbidity [5]. Many anesthetic techniques are used, ranging from sedation to general anesthesia. The worldwide accepted method is deep sedation in the presence of an anesthetist without intubation. Intubation is recommended in very exceptional cases.

Sedation is the most practiced anesthetic technique in most published series [6]. Propofol is the most used drug in this indication $[5,15]$. It reduces procedure time and improves the level of comfort and intra-operative amnesia [5]. Several methods of administration of the product have been evaluated. Intermittent bolus injection remains the most practiced method [16], in addition to sedation controlled by the patient $[7,17]$ and the target controlled infusion [18-20].

ERCP is an uncomfortable procedure requiring adequate sedation or general anesthesia. The required level of sedation during these procedures is often deep. The patient cooperation is an imperative factor for the success of the procedure especially, to avoid intra-operative complications such as duodenal perforations. This deep sedation may compromise the safety of the upper airways and be a source of complications, especially respiratory. Desaturation remains the most observed adverse event $[14,15,21,22]$. 
Table 2 Intraprocedure and recovery room event data

\begin{tabular}{lccc}
\hline Parameter & Group SA $(\mathrm{n}=45)$ & Group TCI $(\mathrm{n}=45)$ & P \\
\hline Procedure time (min) & $88.6 \pm 18.2$ & $85.02 \pm 17.3$ & 0.091 \\
Anesthesia time (min) & $122.4 \pm 16.9$ & $103.7 \pm 18.9$ & $<0.001$ \\
Hypertension episode (yes/no) (n) & $16 / 29$ & $4 / 41$ & 0.014 \\
Hypotension episode (yes/no) (n) & $15 / 30$ & $9 / 36$ & 0.009 \\
Antispasmodique (yes/no) (n) & $19 / 25$ & $15 \pm 2.6$ & 0.023 \\
Extubation time (min) & $27.4 \pm 7.1$ & $40.2 \pm 7.3$ & $<0.001$ \\
Recovery room stay (min) & $58.7 \pm 12.4$ & $1 / 44$ & $<0.001$ \\
Desaturation (yes /No) (n) & $6 / 39$ & & 0.049 \\
Endoscopist satisfaction & & 6 & 0.003 \\
\hline Low & 10 & 13 & 26 \\
\hline Medium & 25 & 10 & \\
\hline High & & & \\
\hline
\end{tabular}

Variables are expressed as mean \pm standard deviation or number.

SA, standard anesthesia; TCI, target-controlled infusion

During sedation for ERCP, several factors may cause desaturation such as advanced age, associated cardiopulmonary co-morbidity, depth of sedation, procedure duration, and the patient's position [12]. This desaturation may be secondary to respiratory depression or inhalation. It is the leading cause of perioperative mortality from cardiovascular complications by causing arrhythmias, myocardial infarction and cardiac arrest, especially in patients with a history of heart disease [14].

The short duration of some interventions, the low incidence of complications, and intubation requirement, associated with the low cost of the procedure are strong arguments for supporters of sedation. The optimization of sedation technique involves choosing an adequate monitoring including oxygenation, depth of anesthesia and especially capnography [23,24].

According to some authors, general anesthesia is less used as an anesthetic technique in ERCP [12]: its major limitation remain the short duration of ERCP, the risk of residual neuromuscular blockade and the high cost. Respiratory and cardiovascular complications are commonly observed with sedation compared to general anesthesia. In a retrospective analysis of 650 procedures, in which 367 were done under sedation, and 238 under general anesthesia, the sedation group patients had significantly more respiratory complications than the general anesthesia group with 13 cases of desaturation requiring mask ventilation in two patients and tracheal intubation in two other patients. In the general anesthesia group, only one patient required a prolongation of mechanical ventilation [12]. In our study, we opted for tracheal intubation in all patients because of the use of muscle relaxants and the need to change patient position. The use of the gastro-laryngeal tube, reported in small series is not without risk as patients may suffer from inhalation, dysphonia and dysphagia $[25,26]$.

To reduce the procedure duration, some authors opt for handiest drugs and using a target-controlled anesthesia mode that allows a shortening of the duration of anesthesia $[19,20]$. TCI is a technique based on the intravenous anesthetic drug infusion device controlled by a software integrated in the device, the infusion rate calculations are based on pharmacokinetic modeling previously validated $[27,28]$. The adjustment of the dose of anesthetic drugs administered is done according to desired concentrations in the brain or the plasma. Propofol, remifentanil and sufentanil are still the most used drugs by this administration mode $[28,29]$. This technique is increasingly used by different centers. However, some authors concluded that further efforts are still needed regarding the availability of devices, knowledge and control of this technique by anesthesiologists [30].

TCI, compared to bolus administration technique or continuous infusion, provides a stable anesthesia level. This drug's titration offers a better control of anesthesia, the maintaining of spontaneous ventilation, hemodynamic stability and a better recovery [20]. Therefore, sedation in spontaneous ventilation and anesthesia in elderly and fragile patients represents the main indications of TCI [28]. In fact, when comparing a standard anesthesia protocol with continuous infusion of propofol and remifentanil to an anesthesia procedure with propofol in TCI mode and continuous infusion remifentanil in a series of 338 patients, undergoing coronary surgery, the authors showed that the tracheal extubation was earlier in the TCI group, but patients in this group required more transfusion and vaso-active drugs [31]. In another prospective study that compares two modes of administration (manual and TCI) of propofol and alfentanil, the authors randomized 220 patients undergoing sedation for gastrointestinal endoscopy. In this study, the recovery time is shorter in the TCI group with less hypotensive episodes and bradypnea. In addition, the risk of desaturation is reduced by $50 \%$ in TCI group [32]. In our study, the recovery time was shorter in patients receiving intravenous anesthesia with target concentrations compared to patients undergoing standard anesthesia. The reduced use of antispasmodics in the TCI group could be explained by the small effect of 


\section{Summary Box}

\section{What is already known:}

- Endoscopic retrograde cholangiopancreatography (ERCP) is the gold standard in the diagnosis and treatment of biliary and pancreatic disease

- Deep sedation without intubation is the most practice anesthetic technique and intubation is recommended in very exceptional cases

- Propofol is the most used drug for sedation

\section{What the new findings are:}

- For patients undergoing an ERCP requiring general anesthesia with intubation, targetcontrolled infusion allows shorter extubation time, more respiratory and hemodynamic stability, and better satisfaction of the endoscopic team than standard anesthesia

remifentanil on the sphincter of Oddi. Compared to morphine, meperidine and remifentanil, it causes less contraction of the Oddi's sphincter [33].

TCI limitation is explained by the fact that it is based on the predicted and not the real concentrations in the plasma or brain [29]. To overcome this limitation, the use of monitoring of the anesthesia depth by the bi-spectral index (BIS) for propofol could optimize these concentrations to the specific needs of each patient $[34,36]$. Some authors reported that closed-loop administration of propofol with TCI, using BIS optimizes this technique $[37,38]$.

ERCP is for a high anesthetic risk population; all efforts must be used to reduce the peri-operative risk. Others factors may influence this risk such as: the experience of the anesthetic team, the anesthesiologists or nurses practicing the anesthesia and the operating room [39]. In a retrospective study, analyzing 1167 sedated patients for ERCP, the authors showed that the experience of the anesthetic team is associated with a lower incidence of desaturation, use of endotracheal intubation and a lower cost of the procedure [40]. Patients were transferred to the gastroenterology or surgery department for their postoperative stay. Postoperative morbidity was not analyzed in this study.

In conclusion, the use of TCI mode allows a better optimization of general anesthesia with short extubation time, decreased need for antispasmodics and less peri-operative complications compared to standard anesthetic technique.

\section{References}

1. Shah SK, Mutignani M, Costamagna G. Therapeutic biliary endoscopy. Endoscopy 2002;34:43-53.

2. Servin F. Anesthésie pour endoscopie digestive. In: Sfar editor.
Conférence d'actualisation. $53^{\mathrm{e}}$ Congrès national d'anesthésie et de réanimation. Paris: Elsevier; 2011.

3. Siegel JH, Kasmin FE. Biliary tract diseases in the elderly: management and outcomes. Gut 1997;41:433-435.

4. Martindale SJ. Anaesthetic considerations during endoscopic retrograde cholangiopancreatography. Anaesth Intensive Care 2006;34:475-480.

5. Kapoor H. Anaesthesia for endoscopic retrograde cholangiopancreatography. Acta Anaesthesiol Scand 2011;55:918-926.

6. Garewal D, Vele L, Waikar P. Anaesthetic considerations for endoscopic retrograde cholangio-pancreatography procedures. Curr Opin Anaesthesiol 2013;26:475-480.

7. Mazanikov M, Udd M, Kylänpää L, et al. Patient-controlled sedation with propofol and remifentanil for ERCP: a randomized, controlledstudy. Gastrointest Endosc 2011;73:260-266.

8. Froehlich F, Schwizer W, Thorens J, Köhler M, Gonvers JJ, Fried M. Conscious sedation for gastroscopy: patient tolerance and cardiorespiratory parameters. Gastroenterology 1995;108:697-704.

9. Chainaki IG, Manolaraki MM, Paspatis GA. Deep sedation for ERCP. World J Gastrointest Endosc 2011;3:34-39.

10. Freeman ML. Adverse outcomes of ERCP. Gastrointest Endosc 2002;56(6 Suppl):S273-S282.

11. Baillie J. Complications of ERCP. En: Jacobson I. ERCP and its applications. 1st ed. Philadelphia-New York: Lippincot-Raven Publishers; 1998, p. 37-54.

12. Sorser SA, Fan DS, Tommolino EE, et al. Complications of ERCP in patients undergoing general anesthesia versus MAC. Dig Dis Sci 2014;59:696-697.

13. Raksakietisak M. Unrecognised aspiration pneumonitis during enteroscopy: two cases report. J Med Assoc Thai 2009;92:869-871.

14. Berzin TM, Sanaka S, Barnett SR, et al. A prospective assessment of sedation-related adverse events and patient and endoscopist satisfaction in ERCP with anesthesiologist-administered sedation. Gastrointest Endosc 2011;73:710-717.

15. Chen WX, Lin HJ, Zhang WF, et al. Sedation and safety of propofol for therapeutic endoscopic retrograde cholangiopancreatography. Hepatobiliary Pancreat Dis Int 2005;4:437-440.

16. Riphaus A, Wehrmann T, Weber B, et al. S3-guidelines-sedation in gastrointestinal endoscopy. Z Gastroenterol 2008;46:1298-1330.

17. Jowell PS, Eisen G, Onken J, Bute BP, Ginsberg B. Patientcontrolled analgesia for conscious sedation during endoscopic retrograde cholangiopancreatography: a randomized controlled trial. Gastrointest Endosc 1996;43:490-494.

18. Mazanikov $M$, Udd $M$, Kylänpää $L$, et al. A randomized comparaison of target-controlled propofol infusion and patientcontrolled sedation during ERCP. Endoscopy 2013;45:915-919.

19. Gillham MJ, Hutchinson RC, Carter R, Kenny GN. Patientmaintained sedation for ERCP with a target controlled infusion of propofol: a pilot study. Gastrointest Endosc 2001;54:14-17.

20. Fanti L, Agostoni M, Casati A, et al. Target-Controlled propofol infusion during monitored anesthesia in patients undergoing ERCP. Gastrointest Endosc 2004;60:361-366.

21. Kongkam P, Rerknimitr R, Punyathavorn S, et al. Propofol infusion versus intermittent meperidine and midazolam injection for conscious sedation in ERCP. $J J$ Gastrointestin Liver Dis 2008; 17:291-297.

22. Goudra B, Singh P, Sinha A. Outpatient endoscopic retrograde cholangiopancreatography: safety and efficacy of anesthetic management with a natural airway in 653 consecutive procedures. Saudi J Anaesth 2013;7:259-265.

23. Lichtenstein DR, Jagannath S, Baron TH, et al. Sedation and anesthesia in GI endoscopy. Standards of Practice Committee of the American Society for Gastrointestinal Endoscopy. Gastrointest Endosc 2008;68:815-826.

24. Weaver J. The latest ASA mandate: $\mathrm{CO}(2)$ monitoring for moderate 
and deep sedation. Anesth Prog 2011;58:111-112.

25. Fabbri C, Luigiano C, Cennamo V, et al. The Gastro-Laryngeal Tube for interventional endoscopic biliopancreatic procedures in anesthetized patients. Endoscopy 2012;44:1051-1054.

26. Gaitini LA, Lavi A, Stermer E, Charco Mora P, Pott LM, Vaida SJ. Gastro-laryngeal tube for endoscopic retrograde cholangiopancreatography: a preliminary report. Anaesthesia 2010;65:1114-1118.

27. Billard V, Cazalaà JB, Servin F, Viviand X. Target-controlled intravenous anesthesia. [Article in French]. Ann Fr Anesth Reanim 1997; 16:250-273.

28. Billard V. Target controlled infusion of opioid in clinical practice. [Article in French]. Le Praticien en anesthésie réanimation 2009; 13:34-40.

29. Billard V. Anesthésie à objectif de concentration intraveineuse (AIVOC) et inhalée (AINOC). In: Sfar, editor. Conférences d'actualisation. 56 e Congrès national d’anesthésie et de réanimation. Paris: Elsevier; 2014.

30. Bertarrex A, Billard V, Molliex S, Passot S, Sztark F. Anesthésie intraveineuse à objectif de concentration (AIVOC): enquête nationale2013.AnnFrAnesthRéanim 2014;33(Suppl.2):A142-A143.

31. Gardellin M, Durand M, Maitrasse B, et al. Continuous infusion of remifentanil and target-controlled infusion of propofol for coronarysurgery in elderly patients: Comparaison with continous infusion of remifentanil and propofol. [Article in French]. Ann Fr Anesth Reanim 2004;23:966-972.

32. Chiang MH, Wu SC, You CH, et al. Target-controlled infusion vs. manually controlled infusion of propofol with alfentanil for bidirectional endoscopy: a randomized controlled trial. Endoscopy
2013;45:907-914.

33. Fragen RJ1, Vilich F, Spies SM, Erwin WD. The effect of remifentanil on biliary tract drainage into the duodenum. Anesth Analg 1999;89:1561-1564.

34. Mahli A, Coskun D, Karaca GI, Akcali DT, Karabiyik L, Karadenizli Y. Target-controlled infusion of remifentanil with propofol or desflurane under bispectral index guidance: quality of anesthesia and recovery profile. J Res Med Sci 2011;16:611-620.

35. Paspatis GA, Chainaki I, Manolaraki MM, et al. Efficacy of bispectral index monitoring as an adjunct to propofol deep sedation for ERCP: a randomized controlled trial. Endoscopy 2009;41:1046-1051.

36. Gruenewald M, Ilies C, Herz J, et al. Influence of nociceptive stimulation on analgesia nociception index (ANI) during propofolremifentanil anaesthesia. Br J Anaesth 2013;110:1024-1030.

37. Liu N, Chazot T, Hamada S, et al. Closed-loop administration of propofol and remifentanil guided by bispectral index: a randomized multicenter study. Anesth Analg 2011;112:546-557.

38. Liu N, Le Guen M, Benabbes-Lambert F, et al. Feasibility of closedloop titration of propofol and remifentanil guided by the spectral M-Entropy monitor. Anesthesiology 2012;116:286-295.

39. Dumonceau JM, Riphaus A, Aparicio JR, et al. European Society of Gastrointestinal Endoscopy, European Society of Gastroenterology and Endoscopy Nurses and Associates, and the European Society of Anaesthesiology Guidelines: Non-anesthesiologist administration of propofol of GI endoscopy. Endoscopy 2010;42:960-974.

40. Goudra BG, Singh PM, Sinha AC. Anesthesia for ERCP: impact of anesthesiologist's experience on outcome and cost. Anesthesiol Res Pract 2013;2013:570518. 